\title{
Heads or Tails? Network Effects on Game Purchase Behavior in The Long Tail Market
}

\author{
Irfan Kanat \\ College of Business \\ Ohio University \\ Athens, Ohio
}

\author{
T.S. Raghu \\ W.P. Carey School of Business \\ Arizona State University \\ Tempe, Arizona
}

\author{
Ajay Vinze \\ Robert J. Trulaske, Sr. College of Business \\ University of Missouri \\ Columbia, Missouri
}

\begin{abstract}
This study aims to uncover the effects of network effects, namely the social influence and network externalities, on purchasing of digital goods. We are particularly interested in the differences in network effects in long tail versus the head of the market. We used a novel dataset from an online game distribution platform covering 1975 games and 8000 users in this study. The results reveal that network effects are more pronounced for the tail of the market in comparison to the head of the market. For the games that are in the head of the market the popularity may be more significantly influenced by factors unobserved in this study (such as advertising budget) yet the games in the tail benefited greatly from any change to the network effects. The exception was social games. The games that relied on user to user engagement did not fare well in the tail of the market. This study contributes to the literature on network effects and long tail market literature by highlighting the differential effects in different segments of the market.
\end{abstract}

Keywords-Online Games; Network Externalities; Social Influence; Digital Economy;

\section{INTRODUCTION}

... the emerging digital entertainment economy is going to be radically different from today's mass market. If the twentieth-century entertainment industry was about hits, the twenty-first will be equally about niches.

Chris Anderson [1]

Software markets are moving towards highly heterogeneous consumer oriented contexts. As a result, users today have greater freedom in choosing software. There have long been calls in the IS community to switch from the traditional mandatory IS settings to more voluntary use contexts [2].
With the shift in the software markets, this need is more salient than ever. Especially in consumeroriented software markets, the decision making responsibility has shifted to the consumers. In these new market settings, characterized by the plethora of choices catering to the wide array of heterogeneous user preferences, choice and subsequent purchasing behavior becomes highly salient.

Prior work has illustrated that freedom of choice has a significant effect on user decision making [3]. While there is a significant body of work on consumer choice in related disciplines, the IS research has only recently begun addressing how consumers make software choices in the presence of alternatives [3]-[5]. Due to unique characteristics of software product -such as, software licenses and susceptibility to network effects- there are opportunities for theory building and testing new boundaries for existing theories. Software products are more susceptible to social influence than most traditional products and goods [6]. With this as a starting point, we aim to better understand the role of social influence and network externalities in software purchasing decision setting.

A software product is defined by the context created by the software itself [7]. Network and brand effects are two dimensions of this self created context. The software is affected by network of users surrounding it and its own brand perceptions. Network effects can be considered in two broad categories: Influence of a user's immediate network is called social influence [8]-[10], and ef- 
fect of the overall network on users' enjoyment is called network externalities [11], [12]. Developers can alter the design of their software to increase the effect of network effects through features that enable user to user interactions [13]-[15]. Here we specifically focus on the moderating role of social features on network effects. Our aim is to answer the following research questions: (1) to what extent do network effects influence purchasing propensity of consumers? (2) Do social features moderate network effects? (3) Are these effects uniform across the market? In answering these questions we take into account the nature of the market and the products by controlling for the brand loyalty and long tail effects.

The digital games are especially suited to study end-user purchasing behavior. Games form a sizable portion of digital market places as entertainment products are one of the most demanded product categories [16]. Another advantage of game market is that the consumer decision making is mainly driven by personal choice and external factors such as business requirements do not influence the choice, hence games provide a clear look at motivations behind purchasing decisions.

To obtain the data used in this study, we developed and deployed a set of Python scripts on amazon web services cloud. We choose a leading online games distribution platform, Steam, for data collection. Steam currently controls $70 \%$ of the digital gaming distribution market, and has over 125 million active users [17], [18]. The data was analyzed with conditional logit model. Our most significant finding is revealing differences in the effectiveness of network effects in the head and tail of the long tail market. We found that the products in the head of the market relied on external factors rather than network externalities or social influence. Still, effect of network externalities were enhanced products with social features in this segment. Conversely, the products in the tail with social features saw a dampening of the effect of their social features. No such interaction was observed for social influence. Meaning social features act upon network externalities and not on social influence.
This study contributes to the IS literature in a few significant ways; (1) We study the purchasing behavior with secondary data from one of the largest online software markets, (2) We investigate the relation between components of network effects, namely the social influence and network externalities, and social features. (3) In this study we reveal differences in segments of the long tail market with regards to the effect of network externalities and social influence.

The paper is structured as follows: We present a review of relevant literature in Section II. The methods used in data collection and analysis are discussed in Section III. Next, the results are presented. Finally the paper is concluded with a discussion of results in Section VI.

\section{LITERATURE REVIEW}

The social judgment theory, the idea of influencing judgments through social processes later gave rise to the literature on social influence [8], [9]. The basic premise of social influence is, that people we are close with influence our decisions. IS literature has investigated social influence in both acceptance research [19], [20] and in social network studies [10].

While psychology literature focused on social influence, economics literature has developed a related but distinct concept, network externalities. Instead of looking at effects of social structure, the economics literature took on a more utilitarian approach and investigated marginal impact of an additional user on utility of all others [21]. Network effects can operate through social influence of close peers or through network externalities. IS literature has identified certain features, such as compatibility of file formats and user interfaces, as able to alter the effects of network effects [12], [13], [22]. There is paucity of evidence as to how software design influences the effects of network effects. Evidence is mostly from analytical studies and empirical evidence is sparse [15], [22], [23].

Software as a product is highly susceptible to network externalities [12]. In case of network externalities, the utility of each user is a function of the user base of the product [15]. Let us assume 
that in absence of externalities, the utility of each product is $U=V-C$. Where $V$ is the intrinsic benefit obtained from the product, and $C$ is the cost of acquiring product. The network externality is such that, the more users use the product, the greater the benefit. Hence, in presence of externalities the utility would be: $U=V(\gamma \theta)-C$, where $\gamma$ is the intensity of network externalities, and $\theta$ is the user base of the product.IS literature has a long history of using certain features -such as file format compatibility, UI similarity, collaborative editing, multiplayer- as proxy to estimate network effects [12], [13]. While these features serve as useful if not perfect proxies for network effects, in reality they enable and enhance network effects through social interactions and utility spillovers of network externalities [22]. The intensity of the network externalities can be engineered through careful design [22]. The social features that facilitate user interactions will increase $\gamma$, hence increasing overall utility derived from the user base $\theta$.

Unlike network externality effects, social influence works through direct links between users [24]. So unlike the size of the user base, the structure of the network comes into play for social influence. The immediate network of others a user is connected to, gives the user a chance to learn from others' behavior [10], [25]. Products that have features that facilitate interactions between users will increase the chances to observe others. Hence, social features can enhance the effect of social influence.

It is crucial to note the long tail phenomenon in any analysis of digital market places [14], [26], [27]. While a few major hits still account for majority of sales, the niche products also find an audience in these digital markets. The literature argues this is due to unlimited shelf space on the supply side and reduced search costs on the demand side [28]. Still the market dynamics are quite different between the tail and the head. While the head enjoys high sales, the only justification for existence of the tail is reduced costs of carrying these items [26]. This increase in variety allows users with unusual tastes to easily access niche content in long tail markets. Empirical evaluation of the phenomenon focused on validating the existence of long tail phenomenon. [14] in an investigation of online and catalog sales of women's clothing found that online channels exhibited less concentration of sales in the top half of the market. Similar results have also been reported for information goods in the context of movie rentals [29]. While long tail markets allow niche products to survive in the tail, the niche products still constitute a much smaller fraction of total sales [14], [29]. Given the differences in network size and structure between the super stars in the head and niche products in the tail, the question of network effects in long tail market merits further inquiry.

Brand loyalty is another factor that IS literature is just beginning to investigate. We control for brand effects in this study to prevent biases. Brand loyalty manifests itself when consumers exhibit a bias towards one or more alternative brands in their purchases [30]. IS research on brand loyalty traditionally focused on the effect of information systems -such as online forums or social media- on brand loyalty and not on effect of brand loyalty on information goods purchases [31], [32]. Switching costs, that are familiar to IS researchers, have been identified as a mechanism used to ensure loyalty, but loyalty is a broader concept [33]. Obviously, the branding of an information good which provides an experience to the user, will be different from more traditional goods such as tooth paste or cars. The consumption of information goods are most often not repeat purchases of same product [34], but purchase of an improved version down the line. Given the unique nature of information goods, it is crucial to control for this important factor.

\section{DATA AND Methods}

\section{A. Data}

The data used in this study comes from Steam, which according to Forbes controls $70 \%$ of the market for downloadable PC games [18], with over 125 Million active users [17]. To collect this data we developed and deployed python scripts 
to scrape public profile pages of 300 randomly selected users and their friends. Figure 1 provides an example of said profile pages. The data we scraped from these profile pages were entirely populated by the Steam and not the user, increasing reliability. In selecting the active users the criteria used was: (1) that they logged in to the steam in the last two months, and (2) had at least one friend. This resulted in tracking a network of 8000 users with 1975 distinct games. We not only obtained data on the friendship network but also the ownership of the games by these users. We used this data to obtain the ownership information used as the dependent variable and the friendship information used in social influence estimation. The average user owned about 39 games.

We obtained the market share of these games via a separate script. This script randomly sampled 200,000 users and obtained a list of games they owned. Due to private profiles and deactivated accounts, we were able to obtain data from around 165,000 users. In estimating the proportion of users in a given population, the statistical power is calculated as $n=\left(\frac{z_{(\alpha / 2)}}{E}\right)^{2}(p(1-p))$. Where, $\mathrm{E}$ is the error margin, $\mathrm{z}$ is the $\mathrm{Z}$ score for given alpha level, and finally $\mathrm{p}$ is the proportion of the real sample ( $p=0.5$ gives the most conservative estimate). The sample size chosen gives us sufficient power to estimate each games' market share with a margin of error less than 0.0031 at alpha level 0.01 . We used data from this script to estimate the user base used as proxy for network externality effects.

The final bit of information came from a third script used to crawl the Steam Store pages of the 1975 games identified in the first step. Information on specific games such as name, developer, features (multiplayer, single player, etc.) was obtained. We used this information to generate variables for social features (multiplayer games) and the brand variables.

Given the procedure above, you can see the list of variables used in this study with their brief descriptions in Table I

Table II shows the descriptive statistics for the variables used in the study. As can be seen, most games have less than 1 percent market share. The different sample sizes reported are due to nature of the variables. Some variables are at user-game pairing level and others are at game level, hence the difference.

\section{B. Analysis Method}

Considering the binary nature of our dependent variable (ownership) and repeated observations of same individual, we used a conditional logit model. The conditional logit model takes into account the common variance due to multiple observations of the same user [36]. The conditional logit is a logit model where utility of each purchase is specified as $n_{i j}=z_{j} / \gamma . z_{j}$ represents a vector of characteristics of the game $j$. Then the purchase propensity is given by the logit specification:

$$
\begin{aligned}
& \pi_{i j}=\frac{e^{n_{i j}}}{1+e^{n_{i j}}} \\
& \text { IV. RESULTS }
\end{aligned}
$$

Due to network size and structure differences between products in the head and the tail of the long tail market, there is reason to believe differences between head and tail of the long tail market [14]. Hence we conducted a split sample analysis for games at the head, games at the tail and games in between. Investigating the distribution of user base figures, we split the sample by their user base numbers. Top 5\% was categorized as the games in the head, $20 \%$ as the games in between, and remaining $75 \%$ as the games in the tail.

Large sample analyses are usually easier to achieve statistical significance. Therefore, based on the suggestions from the recent IS literature [37], we also assess the economic significance of the key determinants using their estimated effect sizes. We see a trend in the effects of our variables of interest. The effects of all variables of interest were lower for the products in the head, and increased in the tail. Each unit change in number of friends owning the game created a $2 \%$ change in purchase odds in the head, $8 \%$ in the middle, and $15 \%$ change in the tail of the market. The results were even more dramatic for network externalities, while user base had no main effect in the head, in 


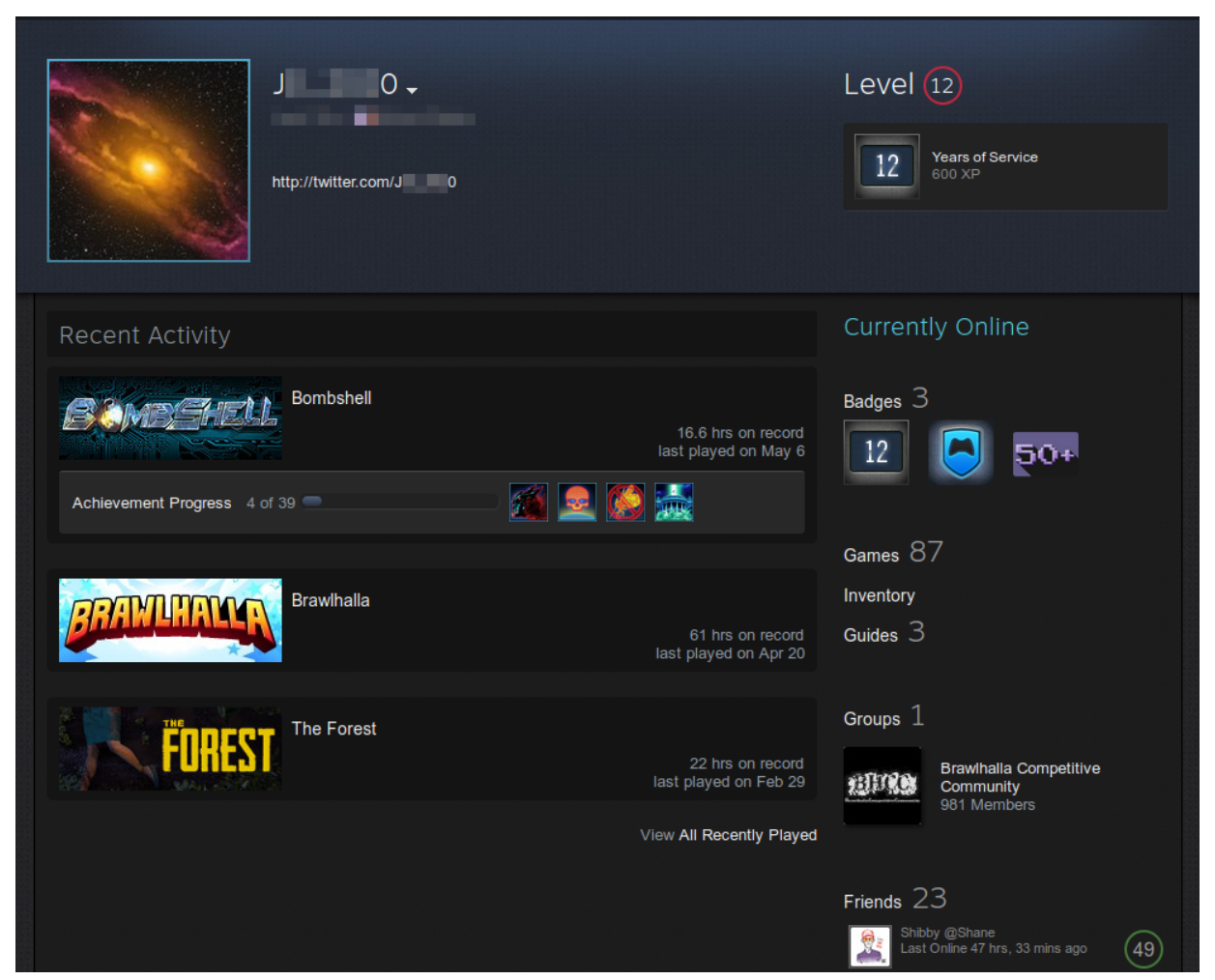

Figure 1. A steam profile page

\begin{tabular}{ll} 
Variables & Description \\
\hline \hline Owned $_{i j}$ & Binary variable describing ownership of game j for user i. \\
FriendsOwn $_{i j}$ & Number of friends owning the same game $\mathrm{j}$ with the user $\mathrm{i}$. \\
DevOwn $_{i j}$ & Number of games from the same developer as game j owned by user i minus 1. \\
Social $_{j}$ & Binary variable describing existence of multiplayer features in game j. \\
Base $_{j}$ & Market share percentage of the game j, a proxy for network externalities. \\
free $_{j}$ & Binary variable for game, 0 if paid, 1 if free. \\
\hline
\end{tabular}

Table I

VARIABLES USED IN THE STUDY.

the middle of the market each point increase in the user base meant $40 \%$ increase in odds, and in the tail each point increase in user base lead to over 300 times increase in odds. These results should be considered in the light of the nature of user base. The user base variable ranges between 0 and 15 , with the mean at 0.089 . A 1 point increase in user base basically makes any game a super star (from 0 to head of the market). Hence the apparent extremity of these results are normal.

Table III reveals a similar pattern in brand loy- alty as well. Overall, we found that when individuals owned another game from the same developer they were $6 \%$ more likely to also own the focal game. We ran robustness checks with alternative measures of brand loyalty and observed similar effect sizes for IP and Series loyalty as well. We can attribute the trend of increasing effects towards the tail to the demand for these games being driven more by external factors such as advertising. Hence the purchasing propensity for the games in the head are not as heavily influenced 


\begin{tabular}{lrrrrrrrr} 
Variable & $\mathbf{n}$ & Min & $\mathbf{q}_{\mathbf{1}}$ & $\widetilde{\mathbf{x}}$ & $\overline{\mathbf{x}}$ & $\mathbf{q}_{\mathbf{3}}$ & $\mathbf{M a x}$ & $\mathbf{s}$ \\
\hline \hline Owned & 590934 & 0 & 0.000 & 0.000 & 0.019 & 0.000 & 1.000 & 0.138 \\
FriendsOwn & 590934 & 0 & 0.000 & 0.000 & 0.640 & 0.000 & 245.000 & 2.992 \\
Social & 590934 & 0 & 0.000 & 0.000 & 0.376 & 1.000 & 1.000 & 0.484 \\
DevOwn & 590934 & 0 & 0.000 & 0.000 & 0.039 & 0.000 & 26.000 & 0.652 \\
Base & 590934 & 0 & 0.037 & 0.089 & 0.251 & 0.222 & 14.934 & 0.650 \\
free & 590934 & 0 & 0.000 & 0.000 & 0.076 & 0.000 & 1.000 & 0.264 \\
\hline
\end{tabular}

Table II

Descriptive Statistics of Revealed Choice Data

\begin{tabular}{lcccc}
\hline & Pooled & Head & Middle & Tail \\
\hline FriendsOwn & $0.022(0.001)^{* * *}$ & $0.021(0.002)^{* * *}$ & $0.081(0.003)^{* * *}$ & $0.143(0.006)^{* * *}$ \\
Base & $0.391(0.015)^{* * *}$ & $0.017(0.026)$ & $0.418(0.106)^{* * *}$ & $5.943(0.338)^{* * *}$ \\
Social & $0.391(0.021)^{* * *}$ & $-0.123(0.072)$ & $-0.082(0.076)$ & $0.036(0.067)$ \\
DevOwn & $0.062(0.003)^{* * *}$ & $0.055(0.004)^{* * *}$ & $0.150(0.005)^{* * *}$ & $0.205(0.005)^{* * *}$ \\
Base x Social & $-0.221(0.015)^{* * *}$ & $0.053(0.026)^{*}$ & $0.001(0.150)$ & $-1.299(0.593)^{*}$ \\
FriendsOwn x Social & $-0.004(0.001)^{* * *}$ & $-0.004(0.002)^{*}$ & $0.002(0.003)$ & $0.005(0.007)$ \\
Free & $-0.020(0.035)$ & $0.058(0.055)$ & $0.059(0.049)$ & $-0.129(0.080)$ \\
\hline AIC & 165719.452 & 25940.020 & 50976.990 & 55254.795 \\
LR Test & $7964(7) p=0$ & $962.2(7) p=0$ & $1380(7) p=0$ & $1865(7) p=0$ \\
Num. events & 11403 & 3018 & 4402 & 3983 \\
Num. obs. & 590936 & 26522 & 120988 & 443126 \\
\hline$* * *<<0.001,{ }^{* *} p<0.01,{ }^{*} p<0.05$ & & &
\end{tabular}

Table III

RESULTS OF ESTIMATION FOR SPLIT SAMPLE ANALYSIS

by network externalities, social influence, or brand loyalty as much as games in the tail end of the market. The games in the tail on the other hand are heavily impacted by other channels such as word of mouth between friends and brand loyalty of existing customers.

For games in the head, we notice that these games only benefit from network externalities when the game had social features. Each point increase in user base meant five percent increase in odds of purchase for multiplayer games in this group. No such effect was observed for single player games in the head of the market. Yet the effect of social features was reversed between the head and tail. Meaning there may be a non-linear relation between social features and user base. If the network is not large enough, as is the case in the tail, the social features actually hurt the game. The reason for this reversal can be summed as: a multiplayer game without players is not attractive.

The interaction between social influence and social features, while statistically significant in the head of the market, was economically insignificant. The net effect was $0.4 \%$ hence quite negligible.

\section{LimitATIONS}

We used a conditional logit model that does not take into account the time effects in this study. The reason for this was due to the nature of our dependent variable. The dependent variable, ownership is binary in nature. Until a person purchases a game, the variable is set to 0 and after purchase it becomes 1. The appropriate model for this kind of relation is a time variant survival model. Yet, survival models focus on a single event (e.g. purchase of a single game). With about 1975 distinct events, the survival model is 
not applicable. While there are risk models that can accomodate a handful of distinct events, we could not find a more appropriate model than a conditional logit model for this study.

Second, the findings presented in this study do not establish causality. Our model used in this study does not account for endogeneity and cyclical correlations between our dependent and independent variables. Hence, we advise the readers against causal reading of the relation between network effects and ownership.

Finally, we would like to remind the readers that brand loyalty in information goods context is slightly different from the pyhsical goods context. We encourage readers to keep this difference in mind in their use of the results.

\section{CONCLUSIONS}

We set out to answer three research questions: (1) understanding the effect of network effects on purchasing behavior, (2) uncovering the role of social features in moderating network effects, and (3) evaluating these effects in different segments of the market. Our findings reveal the network effects and purchasing behavior are related and that the effect of network effects varied between market segments. We found significant differences between the head and the tail of the long tail market in terms of the effectiveness of network effects. The demand at the head of the long tail market was not as influenced by our variables of interest as the tail of the market. This finding suggests that the demand at the head of the market is driven more by factors external to our study such as advertising. It is typical for an AAA game (typical games in the head) to have astronomical advertising budgets. GTA V had a total budget of $\$ 265$ million, out of which $\$ 150$ million was spent for advertising [38].

We found that social influence and network externalities was more effective towards the tail of the market. Lacking the advertising budget of super stars and big name developers, the niche players rely more on user base and social influence to generate sales. These companies can increase their sales through methods that increase their network such as giveaways, free sampling, discounts, and participating in bundles. They can engage and mobilize their existing customers in forums and social media to trigger word of mouth effects. Similarly, we found that brand loyalty was a significant driver in the tail of the market. The smaller firms that operate in the tail of the market, lacking the advertising capabilities, can utilize their reputation and direct contacts with their customers to increase their sales.

In answering our second research question regarding the role of social features, we uncovered an interesting difference between the head and the tail of the market. At the head of the market we found that social features enhanced the effects of network externalities. Meaning when a large user base exists, the social features enhance these effects. We observed opposite effects in the tail of the market. Suggesting if the user base is small social features can have a detrimental role. The games that rely on social features to engage users need a large enough user base to create engagement. In everyday terms, a multiplayer game without players is not very desirable for customers. The literature argues intensity of network effects can be enhanced through product features [15]. Our results reveal a boundary condition in this relationship, if the network size is insufficient these features can harm, rather than enhance utility.

The findings of this study contributes to the literature on social influence and network externalities. We found significant differences in how these effects operated in the head and tail of the long tail market. Our results reveal an important distinction between the workings of the two effects, namely, network externalities can be enhanced through social features. Yet the effect of social influence remains unchanged. Our findings of brand loyalty in software markets, provide empirical evidence for a relation long overlooked by IS research. Future research can expand upon this finding by testing the boundary conditions for this relationship. 


\section{REFERENCES}

[1] C. Anderson, The long tail: Why the future of business is selling less of more. Hachette Books, 2006.

[2] I. Benbasat and H. Barki, "Quo Vadis, TAM?," Journal of the Association for Information Systems, vol. 8, pp. 211-218, Sept. 2007.

[3] K. B. Murray and G. Häubl, "Freedom of Choice, Ease of Use, and the Formation of Interface Preferences.," MIS Quarterly, vol. 35, no. 4, pp. 955-A6, 2011.

[4] A. Schwarz and C. Schwarz, "Incorporating choice into models of technology adoption," in 15th Americas Conference on Information Systems, p. 319, 2009.

[5] A. Schwarz and C. Schwarz, "An exploration of the individual-level post-adoption choice decision," Journal of Information Technology Theory and Application (JITTA), vol. 15, no. 3, p. 2, 2015.

[6] K. J. Stewart, A. P. Ammeter, and L. M. Maruping, "Impacts of License Choice and Organizational Sponsorship on User Interest and Development Activity in Open Source Software Projects," Information Systems Research, vol. 17, pp. 126-144, June 2006.

[7] I. Kanat, Y. Hong, B. Gu, and R. Santanam, "If you let them build it, they will stay! an empirical study of add-on content and user engagement," in Proceedings of the 50th Hawaii International Conference on System Sciences, (Waikoloa, HI), 2017.

[8] M. Sherif and C. I. Hovland, Social judgment: Assimilation and contrast effects in communication and attitude change. Binghamton, NY: Yale Univer. Press, 1961.

[9] G. Robins, P. Pattison, and P. Elliott, "Network models for social influence processes," Psychometrika, vol. 66, no. 2, pp. 161-189, 2001.

[10] P. Singh and C. Phelps, "Networks, social influence and the choice among competing innovations: Insights from open source software licenses," Information Systems Research, vol. 24, no. 3, pp. 539-560, 2009.

[11] J. Farrell and G. Saloner, "Installed base and compatibility: Innovation, product preannouncements, and predation," The American Economic Review, vol. 76, pp. 940-955, 1986.
[12] E. Brynjolfsson and C. F. Kemerer, "Network Externalities in Microcomputer Software: An Econometric Analysis of the Spreadsheet Market," Management Science, vol. 42, no. 12, pp. 1627-1647, 1996.

[13] N. Gandal, "Competing compatibility standards and network externalities in the pc software market," The Review of Economics and Statistics, vol. 77, no. 4, pp. 599-608, 1995.

[14] E. Brynjolfsson, Y. Hu, and D. Simester, "Goodbye pareto principle, hello long tail: The effect of search costs on the concentration of product sales," Management Science, vol. 57, no. 8, pp. 1373-1386, 2011.

[15] H. Cheng, Y. Liu, and Q. Tang, "The Impact of Network Externalities on the Competition Between Open Source and Proprietary Software," Journal of Management Information Systems, vol. 27, no. 4, pp. 201-230, 2011.

[16] AppBrain, "Free vs. paid Android apps," 2016.

[17] S. Sherif, "Steam has over 125 million active users, $8.9 \mathrm{~m}$ concurrent peak." http://www.vg247.com/2015/02/24/ steam-has-over-125-million-active-users-8-9m-concurrent-peak/, February 2015. Accessed: 2015-04-15.

[18] O. Chiang, "The master of online mayhem." http://www.forbes.com/forbes/2011/0228/ technology-gabe-newell-videogames-valve-online-mayhem. html, September 2011. Accessed: 2015-04-15].

[19] V. Venkatesh and F. D. Davis, "A theoretical extension of the technology acceptance model: Four longitudinal field studies," Management science, vol. 46, no. 2, pp. 186-204, 2000.

[20] V. Venkatesh and M. G. Morris, "Why don't men ever stop to ask for directions? gender, social influence, and their role in technology acceptance and usage behavior," MIS quarterly, pp. 115-139, 2000.

[21] M. L. Katz and C. Shapiro, "Network externalities, competition, and compatibility," The American economic review, pp. 424-440, 1985.

[22] Y. Dou, M. F. Niculescu, and D. J. Wu, "Engineering Optimal Network Effects via Social Media Features and Seeding in Markets for Digital Goods and Services," Information Systems Research, vol. 24, pp. 164-185, mar 2013. 
[23] Y. Liu, H. K. Cheng, Q. C. Tang, and E. Eryarsoy, "Optimal software pricing in the presence of piracy and word-of-mouth effect," Decision Support Systems, vol. 51, pp. 99-107, apr 2011.

[24] Z. Katona, P. P. A. L. Zubcsek, and M. Sarvary, "Network Effects and Personal Influences : The Diffusion of an Online Social Network," Journal of Marketing Research, vol. XLVIII, no. June, pp. 425-443, 2011.

[25] D. L. McFadden and K. E. Train, "Consumers' Evaluation of New Products: Learning from Self and Others," Journal of Political Economy, vol. 104, no. 4, pp. 683703, 1996.

[26] C. Anderson, "The long tail," Wired, vol. 12, no. 10, pp. 170-177, 2004.

[27] G. Lee and T. Raghu, "Determinants of mobile apps' success: Evidence from the app store market," Journal of Management Information Systems, vol. 31, no. 2, pp. 133-170, 2014.

[28] E. Brynjolfsson, Y. J. Hu, and M. D. Smith, "From niches to riches: Anatomy of the long tail," Sloan Management Review, vol. 47, no. 4, pp. 67-71, 2006.

[29] A. Zentner, M. Smith, and C. Kaya, "How Video Rental Patterns Change as Consumers Move Online," Management Science, vol. 59, no. June 2015, pp. 2622-2634, 2013.

[30] J. Jacoby and B. Kyner, "Brand Loyalty Vs. Repeat Purchasing," Journal of Marketing Research, vol. 10, no. 1, pp. 1-9, 1973.

[31] R. Shang, Y. Chen, and H. Liao, "The value of participation in virtual consumer communities on brand loyalty," Internet Research, vol. 16, no. 4, pp. 398418, 2006.

[32] M. Laroche, M. R. Habibi, and M.-O. Richard, "To be or not to be in social media: How brand loyalty is affected by social media?," International Journal of Information Management, vol. 33, no. 1, pp. 76-82, 2013.

[33] Z. Yang and R. T. Peterson, "Customer perceived value, satisfaction, and loyalty: The role of switching costs," Psychology and Marketing, vol. 21, no. 10, pp. 799-822, 2004.

[34] P. Nelson, "Information and consumer behavior," The Journal of Political Economy, vol. 78, no. 2, pp. 311329, 1970.
[35] K. Rufibach, "reporttools: R functions to generate LTEX tables of descriptive statistics," Journal of Statistical Software, Code Snippets, vol. 31, no. 1, 2009.

[36] D. McFadden, "Econometric analysis of discrete data," Fisher-Schultz Lecture, 1979.

[37] M. Lin, H. C. Lucas Jr, and G. Shmueli, "Research commentary-too big to fail: Large samples and the pvalue problem," Information Systems Research, vol. 24, no. 4, pp. 906-917, 2013.

[38] R. Fleming, "Grand theft auto 5 has now set seven world records." http://www.digitaltrends.com/gaming/ grand-theft-auto-5-has-now-set-seven-world-records/ \#:tO73rFc_4ScmYA, 2013. Accessed: 2016-05-25.

[39] P. Leifeld, "texreg: Conversion of statistical model output in R to $\mathrm{HT}_{\mathrm{E}} \mathrm{X}$ and HTML tables," Journal of Statistical Software, vol. 55, no. 8, pp. 1-24, 2013. 